\section{Proteases: to each his own}

\section{Dagmar Ringe}

PROCESSING of propeptides involves the cleavage of one or more peptide bonds in the inactive precursor resulting in a smaller, sometimes fragmented, active protein. Very often these processing enzymes are unique to a particular propeptide and possess unusual specificity aimed at a limited number of cleavage sites. A paper on page 768 of this issue ${ }^{1}$, and one earlier this month in Science ${ }^{2}$, describe such a protease whose function is the processing of the propeptide from which the cytokine interleukin-1 $\beta$ (IL$1 \beta)$ is derived. This specific proteolytic activation is part of a control mechanism designed to prevent random or unwanted side reactions, which might damage the cell by the activation of potentially dangerous molecules. Consequently, each activation process is highly specific for its own particular target.

Many proteins are synthesized as inactive precursors. The earliest identified examples belong to the eukaryotic serine protease family of proenzymes. Proteolytic enzymes are usually biosynthesized as somewhat larger inactive precursors known as zymogens, so preventing digestion of the tissues that synthesize them. Zymogens have very low enzymatic activity because the active site is distorted or blocked, preventing the productive binding of substrate. For digestive enzymes, specific tryptic cleavage at an arginine or lysine residue produces active enzyme in the case of trypsin and elastase, or produces an intermediate form which is processed further by autolysis to give active $\alpha$-chymotrypsin. All of this processing takes place in the duodenum in order to protect the pancreas from digestion, a sometimes fatal condition.

The prokaryotes seem to have developed a slightly different mechanism whereby autodigestion is prevented ${ }^{3}$. For instance, $\alpha$-lytic protease is synthesized as a pre-proprotein which is activated autocatalytically. The precursor plays two important roles in the control mechanism, which protects the cell proteins from random attack by this protease. First, the precursor is inactive towards substrates, although it does have some low level of autocatalytic activity. But, more notably, the precursor is required for this protein to fold properly into its final three-dimensional form.

Early attempts to express mutants of this enzyme, designed to probe the functions of active-site residues, were unsuccessful unless the longer protein was expressed. Activation of the precursor is then required to produce the correct form of the final protease. These mutants have little catalytic activity, if any, so this requirement would seem to result in a conundrum. The problem has been solved by identification of the proregion as a folding template which can be used separately to accelerate the formation of the active, native state $^{4}$. Attempts to refold $\alpha$-lytic protease in vitro resulted in an inactive, but foldingcompetent state with native-like secondary structure. Addition of the proregion as a separate polypeptide chain resulted in recovery of active enzyme.

Biogenesis of the proteins and biologically active peptides required as chemi-

\title{
Picture a specific translocation
}
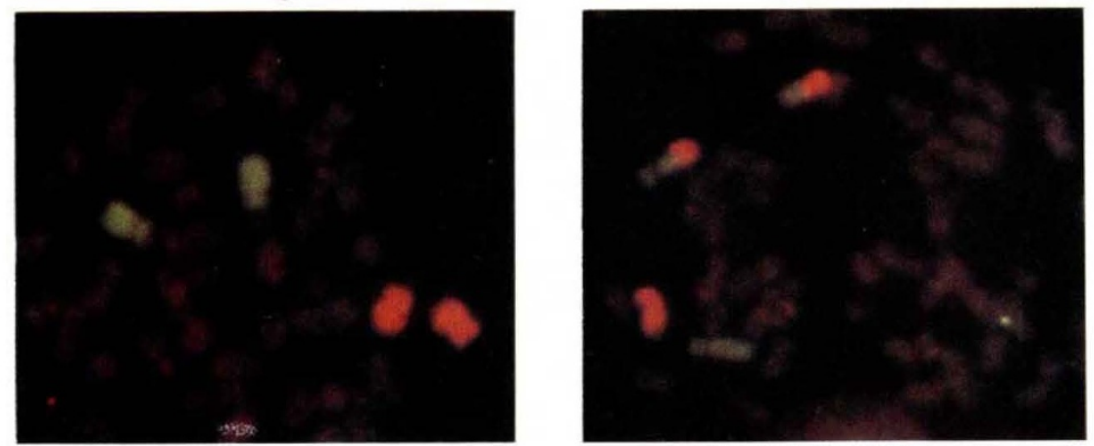

MORE colour means more power In fluorescence in situ hybridization, a technique usually known by Its acronym FISH or as chromosome painting. In Its latest form, three-colour painting, the approach can be used to identify translocations between two speciflc chromosomes as caused, for example, by Ionizing radlation; previously, 'banding' methods, which are slower, were used for the purpose. These two plctures come from the group of J.N. Lucas at Lawrence Livermore Natlonal Luboratory and lliustrate the point. That on the left shows human chromosomes 4 (green) and 12 (red) In normal metaphase; that on the right translocation between the two chromosomes. The third colour involved is a propidlum lodide counterstain.

T.L.

cal messengers for various forms of cellprocess regulation is often regulated by a similar mechanism. Hormones such as insulin or $\beta$-endorphin are synthesized as prohormones or more complicated polyprotein precursors. The identification of the endoprotease Kex 2 as essential for the processing of the precursors of yeast killer toxin and $\alpha$-mating factor provided a clue to the possible nature of the enzymes responsible for processing hormone precursors 5 . From sequence analysis, Kex2 seems to be a subtilisinlike serine protease. Similar enzymes have now been identified in higher eukaryotic systems, which can reproduce all the main cleavages of a pituitary hormone precursor. This polyprotein, proopiomelanocortin, contains a total of seven different polypeptide hormones. Post-translational processing in the different lobes of the pituitary leads to the formation of various hormones with different physiological activities. Because the processing activities are different, different hormones seem to arise from different lobes, although all of them ultimately come from the same precursor. The processing proteases, PC2 and PC1/PC3, are distributed differently between the pituitary lobes, accounting for the distribution of the observed cleavage pattern ${ }^{6}$. The cleavage sites have a unique specificity, in that cleavage occurs between basic residues at Lys-Arg or Lys-Lys sites.

The control of hormone production, then, comes from a complex interplay of compartmentalization of highly specific processing proteases. So why is such a large polyprotein necessary? The answer may lie in a mechanism reminiscent of the $\alpha$-lytic protease story. The specificity of the cleavage sites may be enhanced by secondary and tertiary structure of the polypeptide involved in presentation of cleavage sites to the proper protease.

The existence of a specific protease responsible for the activation of IL-1 $\beta$ would seem to follow naturally from these considerations. Mature IL- $1 \beta$ is a major mediator of inflammation. It is also regarded as a key hormone of the immune system, and has been implicated in septic shock and wound healing ${ }^{7}$. Attempts at control of this messenger and its associated activities have, up to now, centred on antagonists that resemble IL-1 $\beta$ in sequence and structure ${ }^{8}$. Attempts to discover low-molecularweight antagonists which might be capable of blocking the IL- $1 \beta$ receptor without eliciting the normal response, and which would be suitable for oral therapy, have been unsuccessful. The particular importance of the new work reported by Thornberry et al. ${ }^{1}$ and Cerretti et al. ${ }^{2}$ is that it offers a new target for therapeutic intervention.

Interleukin- $1 \beta$ is synthesized as an in- 